\title{
Demographic Profile and Prevalence of Comorbidities in Newly Diagnosed Women with Breast Cancer: Experience from a Tertiary Care Institute
}

\author{
Yeshwanth Sankranthi ${ }^{1 *}$, Neelima Kambhayathghar ${ }^{1}$, Suseela Kodandapani ${ }^{1}$, Nisha Hariharann ${ }^{2}$ \\ Chandra CK Naidu², Raju KVVN ${ }^{2}$, Yugandar Reddy², Rao ST2 and Sudha Murthy S 3 \\ ${ }^{1}$ Department of Clinical Research, Basavatarakam Indo American Cancer Hospital and Research Institute, Hyderabad, Telangana, India \\ ${ }^{2}$ Department of Surgical Oncology, Basavatarakam Indo American Cancer Hospital and Research Institute, Hyderabad, Telangana, India \\ ${ }^{3}$ Department of Laboratory Medicine, Basavatarakam Indo American Cancer Hospital and Research Institute, Hyderabad, Telangana, India
}

*Corresponding author: Yashwanth Sankranthi, Clinical Pharmacologist, Department of Clinical Research, Basavatarakam Indo American Cancer Hospital and Research Institute, Hyderabad, Telangana, India, Tel: +91-9642230230; E-mail: yashwanthsankranthi@gmail.com

Received Date: Nov 26, 2018; Accepted Date: Dec 04, 2018; Published Date: Dec 06, 2018

Citation: Sankranthi Y, Kambhayathghar N, Kodandapani S, Hariharan N, Naidu CCK, et al. (2018) Demographic Profile and Prevalence of Comorbidities in Newly Diagnosed Women with Breast Cancer: Experience from a Tertiary Care Institute. Ann Clin Lab Res Vol.6 No.4:270.

\section{Abstract}

Background: Women with breast cancer comprise a very heterogeneous group. For Indian women, there is evidence to suggest that the age at presentation and demographic profile are different from their Western counterparts. We undertook this study to understand the demographic profile and prevalence of co-morbidities in newly diagnosed women with breast cancer presenting at our institute.

Materials and methods: We conducted an audit of a prospectively maintained database at our breast clinic. All women presenting with a breast lump with available demographic data between $1^{\text {st }}$ June 2017 and $31^{\text {st }}$ October 2017 were included in the study.

Results: Of the 376 women who visited our breast clinic between June to October 2017, 257 were diagnosed to have a breast malignancy. Among these, the mean age at presentation was 51 years. $61.8 \%$ were post-menopausal. The histology in majority of the women was ductal carcinoma (94.1\%). The stage wise distribution was as follows-7.3\% had Stage I disease, $43.1 \%$ had Stage II, $37.3 \%$ had Stage III and $8.1 \%$ presented with metastatic disease. The co-morbidities profile showed $26 \%$ of the women had hypertension and $20.6 \%$ had diabetes mellitus. None of the women had dyslipidaemia. $13.6 \%$ women had both hypertension and diabetes. On evaluation of the risk factors for breast cancer, 4 women gave a history of alcohol consumption, 5 women had a previous history of lumpectomy in the breast, and 4 women had a significant family history of cancer. $40 \%$ of the women were overweight and another $14 \%$ were obese at presentation.

Conclusion: Breast cancer is a heterogeneous disease and Indian women differ significantly from the Western population in the stage at presentation. The risk factors profile shows very few Indian women have an identifiable risk factor (5\%). Also, of the women who present with breast cancer, one in four has associated hypertension and one in five has diabetes mellitus. Further studies are warranted to accurately document the risk factor profile and associated co-morbidities in Indian women.

Keywords: Breast cancer; Co-morbidities; Risk factors

\section{Introduction}

Breast cancer is a diverse and heterogeneous disease affecting women in both the developing and developed countries. According to GLOBOCAN 2012, India recorded 70,218 deaths in 2012 due to breast cancer alone, the maximum in the world followed by China $(47,984)$ [1]. The incidence of breast cancer in India has been rising steadily and has overtaken cervical cancer as the most common cancer in women [2].

In India, although age adjusted incidence rate of breast cancer is lower (25.8 per 100 000) than United Kingdom (95 per 100000$)$ but mortality is at par (12.7 vs. 17.1 per 100000) with United Kingdom [3]. There is a significant increase in the incidence and cancer-associated morbid-ity and mortality in Indian subcontinent as described in global and Indian studies [5-8].

Breast cancer is multi-factorial in etiology. Early menarche, late menopause, delayed pregnancy, lack of breast feeding, family history of cancer and previous lumpectomies are among the various risk factors that have been studied. The reasons for the low incidence of breast cancer among Indian women and the increasing incidence observed in recent years are not completely understood although likely to be explained by reproductive and lifestyle factors. With the fast evolution in socioeconomic status, the lifestyle of women in Indian cities gradually resembles their Western counterparts. Consequently, the prevalence of risk factors for breast cancer is on an increase. 
Lifestyle changes also impact the prevalence of various associated co-morbidities like hypertension, diabetes mellitus, dyslipidaemia and obesity. Concurrent co-morbidities may necessitate a modification in the treatment schedule for breast cancer. Thus, identifying and understanding the prevalence of associated co-morbidities can significantly impact cancer care.

There are considerable variations in the prevalence of risk factors and co-morbidities in women across the world. The information on the epidemiology of breast cancer and associated risk factors is very limited. Knowledge of these factors and the co-morbidities profile can help formulate targeted risk reducing strategies. Hence, we undertook this study with the aim of understanding the demographic details and prevalence of risk factors and co-morbidities among women presenting to our institute.

\section{Materials and Methods}

We conducted an audit of a prospectively maintained database at our breast clinic. All women presenting with a breast lump with available demographic data between $1^{\text {st }}$ June 2017 and $31^{\text {st }}$ October 2017 were screened for the study. Women with histological confirmation of cancer were included in the analysis. Patients with recurrent cancer were excluded. Data was collected from the Electronic Medical Records and Medical Records Department. Demographic data, stage at presentation and prevalence of risk factors and co-morbidities were documented for analysis.

The presence of co-morbidities was ascertained by history from the patient and a responsible attendant. A woman was considered to have hypertension/diabetes/dyslipidaemia if she was currently taking medications or had a past history of medications for the same. Obesity was assessed using the Body Mass Index scale. The Body Mass Index was calculated as weight in kilograms divided by the square of the height in meters. A BMI of 18.5-24.99 was considered as normal. BMI equal to or more than 25 was documented as overweight and women with a BMI above 30 were considered as obese. In women with a family history of cancer, the site of cancer and the relationship to the patient were documented. History of breast or ovarian cancer among first or second degree relatives was considered to be significant. Clinical staging was documented according to the American Joint Committee on Cancer (AJCC) $7^{\text {th }}$ edition staging classification.

Informed consent was obtained from all the participants. This study received approval from our Institutional Ethics Committee.

\section{Results}

Of the 376 women who visited our breast clinic between $1^{\text {st }}$ June 2017 and 31 ${ }^{\text {st }}$ October 2017, 257 were diagnosed to have a malignancy of the breast and were included in the analysis. The mean age at presentation was 51 years (22-83 years). $61.8 \%$ of the women were postmenopausal.

The stage wise distribution at presentation showed one half of the women presented with operable breast cancer $(50.4 \%)$ and $37.3 \%$ presented at a locally advanced stage. $8.1 \%$ were de novo metastatic at presentation. The most common site of metastasis was the bone ( 7 women) followed by visceral metastasis to the lung ( 4 women). 4 women had both bony and visceral metastasis at presentation (Figure 1).

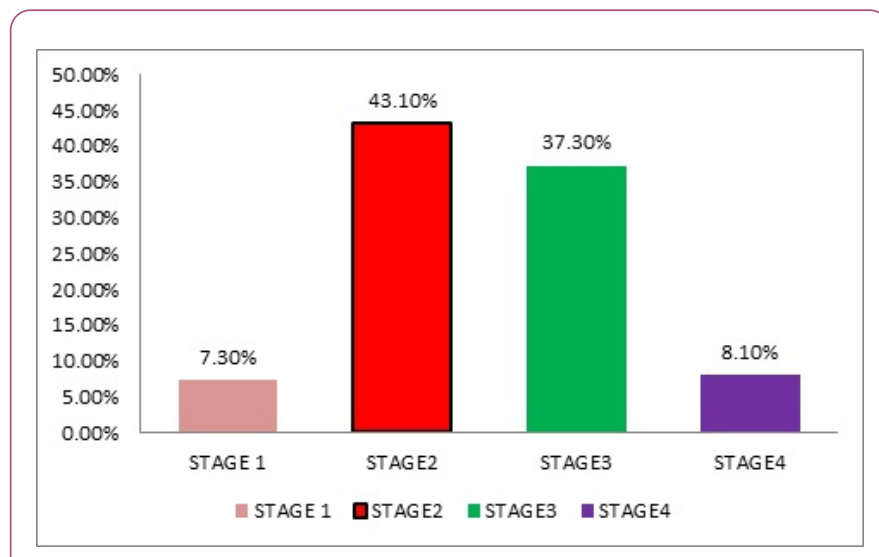

Figure 1 Stage wise distribution at presentation.

The predominant histology was duct carcinoma (94.1\%). $1.9 \%$ of the women presented with phyllodes tumor and another 3.5\% had rare histology's like metaplastic carcinoma or mucinous or papillary carcinoma (Figure 2).

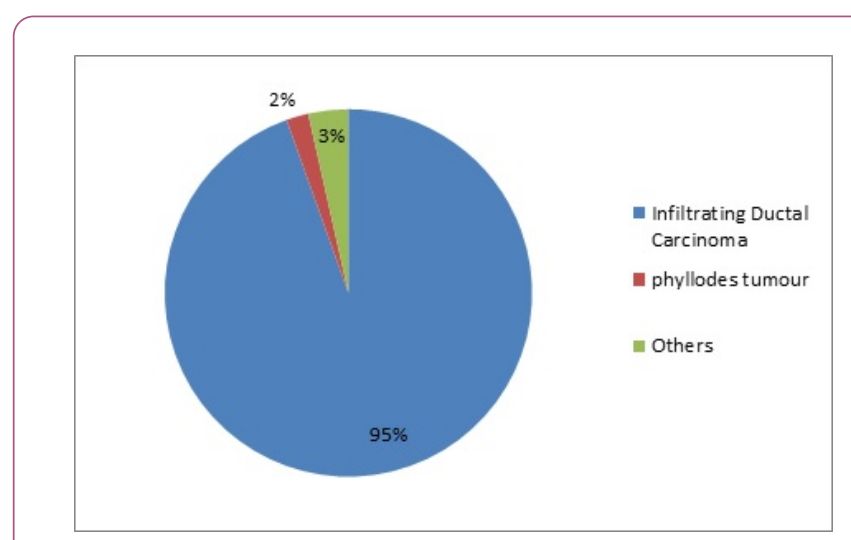

Figure 2 Types of carcinoma.

For the analysis, hypertension, diabetes mellitus and dyslipidaemia were documented as co-morbidities. $26 \%$ of the women were on medications for hypertension and $20.6 \%$ were taking treatment for diabetes mellitus. None of the women in our cohort had a previous history of dyslipidaemia. $13.6 \%$ of the women had both hypertension and diabetes and were on concurrent medications for the same.

Of the risk factors documented in our analysis, history of alcohol addiction was given by 4 women. Another 5 women had a history of a previous lumpectomy in the breast for noncancerous lesions. 4 women $(1.5 \%)$ had a family history of breast cancer. $40 \%$ of the women were overweight and 
another $14 \%$ were obese at presentation. The documented risk factors and co-morbidities are summarized in Table 1.

Table 1 Demographic presentation of study participants, distribution of breast comorbidities and risk factors.

\begin{tabular}{|c|c|c|}
\hline Variables & Frequency & Percentage \\
\hline Age & $\begin{array}{l}\text { Mean age } 51 \\
\text { years }(22-83)\end{array}$ & -- \\
\hline \multicolumn{3}{|l|}{ Menopausal status } \\
\hline Premenopausal & 98 & $38.1 \%$ \\
\hline Postmenopausal & 159 & $61.8 \%$ \\
\hline \multicolumn{3}{|l|}{ Comorbidities } \\
\hline Hypertension (HTN) & 67 & $26 \%$ \\
\hline Diabetes mellitus (DM) & 53 & $20.6 \%$ \\
\hline Dyslipidaemia & None & Nil \\
\hline Both HTN and DM & 35 & $13.6 \%$ \\
\hline \multicolumn{3}{|l|}{ Risk factors } \\
\hline Alcohol addiction & 4 & -- \\
\hline $\begin{array}{l}\text { Previous breast } \\
\text { lumpectomy }\end{array}$ & 5 & -- \\
\hline $\begin{array}{l}\text { Family history of breast } \\
\text { cancer }\end{array}$ & 4 & -- \\
\hline Obesity & -- & -- \\
\hline
\end{tabular}

118 women visited our clinic for routine screening during the same period and were excluded from the above analysis. Among these women, 10 were treated for abscess/ granulomatous mastitis and 15 were diagnosed to have fibro adenomas/fibrocystic disease. Among these 118 women, $18(15.2 \%)$ had a significant family history of cancer.

\section{Discussion}

The data of 376 newly registered women at our breast clinic was evaluated in this study. Of these, 257 were diagnosed with breast cancer and were included in the analysis. The mean age at presentation was 51 years (range $22-83$ years) and $61.8 \%$ were postmenopausal. In India, breast cancer incidence peaks between the ages of 45-50 years as compared to 61 years in the Western literature [9]. In UK in 2012-2014, on average each year almost half (48\%) of cases were diagnosed in people aged 65 and over [10]. Multiple hypotheses have been postulated for this decade earlier presentation. Breast cancer in Indian women is probably biologically different and more aggressive as compared to the Western population and often tends to present at an earlier age.

The stage wise distribution at presentation in our dataset showed one half of the women presented with operable breast cancer $(50.4 \%)$ and $37.3 \%$ presented at a locally advanced stage. $8.1 \%$ women were metastatic at presentation. This is in agreement with the data published by Agarwal et al. [11], where they found almost one half of the patients presenting at a locally advanced stage. Unfortunately, breast cancer remains shrouded by stigma in many Indian societies. This coupled with the lack of education and awareness often leads to women presenting at an advanced stage. Unique to India are the cultural factors and dominance of traditional medicine healers. Lack of access to medical facilities further compounds the problem. This late stage at presentation could partially be responsible for the dismal survival outcomes in India. GLOBOCAN 2012 estimated that 1 in 2 women diagnosed with breast cancer in India, succumb to it [1].

In our cohort, one in four women was hypertensive and one in five women was on treatment for diabetes mellitus. None of our patients gave a history of dyslipidaemia. $13.6 \%$ of the women were on concurrent medications for diabetes and hypertension at the time of diagnosis of cancer. This prevalence of co-morbidities in our cohort correlates with the data that almost one half of our women (54\%) were either overweight or obese at presentation. This data is in agreement with a study done by Singh et al., from north India, where they found $46.1 \%$ of their study subjects to have a normal BMI [12]. Obesity coupled with hypertension and diabetes forms part of the metabolic syndrome. With the changing lifestyle patterns in India, an increasing number of patients diagnosed with breast cancer have one of the above co-morbidities. This can have a significant impact on the tolerance to therapy and on some occasions may even require change in therapy. It is of crucial importance to the treating oncologist to document these co-morbidities and plan therapy accordingly. Appropriate health and diet counselling should be integrated into the cancer care for such patients.

Risk factors such as smoking, alcohol consumption, previous breast biopsies, family history of breast cancer have been well studied and documented. Paksresht et al. [13], in their case control study of risk factors for breast cancer in north India, noticed less than one percent of their women had a history of smoking or alcohol consumption. A combined analysis of 52 epidemiological studies reported $12.9 \%$ of women to have a family history of breast cancer [14]. In our dataset, only $5 \%$ of the women had one of the above documented risk factors and only $1.5 \%$ women had a significant family history of cancer. Agarwal et al., observed that approximately only 5\% of Indian women had a family history of breast or ovarian cancer despite a younger age at presentation [15]. The biology of breast cancer in Indian women seems to be inherently different from their Western counterparts and very few Indian women have associated risk factors at the time of diagnosis.

\section{Conclusion}

Women in India are diagnosed at a later stage at presentation as compared to their Western counterparts. This negatively impacts the outcomes of these women. There is an increasing prevalence of associated co-morbidities among newly diagnosed women with breast cancer. Appropriate addressal of these co-morbidities along with cancer care will become crucial in the management of such women. Risk factors for breast cancer are not common among Indian 
women and it may be an erroneous method of identify high risk women for screening.

\section{Conflicts of Interest}

The authors declare no conflict of interest.

\section{References}

1. Ferlay J, Soerjomataram I, Ervik M, Dikshit R, Eser S, et al. (2012) GLOBOCAN 2012 v1.0, Cancer Incidence and Mortality Worldwide: IARC Cancer Base No. 11.

2. Asthana S, Chauhan S, Labani S (2014) Breast and cervical cancer risk in India: An update. Indian J Public Health 58(1): 5.

3. Gupta A, Shridhar K, Dhillon PK (2015) A review of breast cancer awareness among women in India: Cancer literate or awareness deficit?. Eur J Cancer 51(14): 2058-2066.

4. Porter PL (2009) Global trends in breast cancer incidence and mortality. Salud Pública De México 51: 141-146.

5. Babu GR, Lakshmi SB, Thiyagarajan JA (2013) Epidemiological correlates of breast cancer in South India. Asian Pac J Cancer Prev pp: 5077-5083.

6. Ali I, Wani WA, Saleem K (2011) Cancer scenario in India with future perspectives. Cancer Therapy 8: 56-70.

7. Srinath Reddy K, Shah B, Varghese C, Ramadoss A (2005) Responding to the threat of chronic diseases in India. Lancet 366: 1744-1749.
8. Balasubramaniam S, Rotti S, Vivekanandam S (2013) Risk factors of female breast carcinoma: A case control study at Puducherry. Indian J Cancer 50: 65-70.

9. Leong SP, Shen ZZ, Liu TJ, Agarwal G, Tajima T, et al. (2010) Is breast cancer the same disease in Asian and Western countries?. World J Surg 34(10): 2308-2324.

10. https://www.cancerresearchuk.org/health-professional/cancerstatistics/statistics-by-cancer-type/breast-cancer\#heading-Zero.

11. Agarwal G, Pradeep PV, Aggarwal V, Yip CH, Cheung PS (2007) Spectrum of breast cancer in Asian women. World J Surg 31(5): 1031-1040.

12. Singh $M$, Jangra B (2013) Association between body mass index and risk of breast cancer among females of north India. South Asian J Cancer 2(3): 121.

13. Pakseresht S, Ingle GK, Bahadur AK, Ramteke VK, Singh MM, et al. (2009) Risk factors with breast cancer among women in Delhi. Indian J Cancer 46(2): 132.

14. Collaborative Group on Hormonal Factors in Breast Cancer (2001) Familial breast cancer: Collaborative reanalysis of individual data from 52 epidemiological studies including 58209 women with breast cancer and 101986 women without the disease. The Lancet 358(9291): 1389-1399.

15. Agarwal G, Ramakant P (2008) Breast cancer care in India: The current scenario and the challenges for the future. Breast care 3(1): 21-27. 